\title{
Microscopic Findings Vendor Name
}

National Cancer Institute

\section{Source}

National Cancer Institute. Microscopic Findings Vendor Name. NCI Thesaurus. Code C117590.

The literal identifier of the vendor or laboratory that performs a microscopic assessment. 